\title{
Los extranjerismos crudos en el DRAE 2014: Cambios en el diccionario académico*
}

\author{
CRISTINA FERNÁNDEZ BERNÁRDEZ \\ Universidade da Coruña \\ c.fbernardez@udc.es
}

\section{INTRODUCCIÓN}

En octubre de 2014 ve la luz la vigesimotercera edición del Diccionario de la lengua española $(D R A E)$, trece años después de la publicación de la anterior. Uno de los primeros aspectos que interesa tanto a los estudiosos como a los usuarios habituales de un nuevo diccionario académico es descubrir qué novedades aporta ${ }^{1}$. Algunas de las que presenta el DRAE 2014 se habían ido adelantando, bien en el Diccionario esencial de la lengua española (2006), bien vía electrónica en el avance de la 23. ${ }^{\text {a }}$ edición, disponible hasta hace poco en la página web de la Academia ${ }^{2}$. Hay que tener presente, además, que entre la vigesimosegunda y la vigesimotercera edición del $D R A E$, se han publicado algunas obras académicas fundamentales, como el Diccionario panhispánico de dudas (2005), la Nueva gramática de la lengua española (2009 y 2011) y la Ortografía de la lengua española (2010), todas ellas con repercusiones, evidentemente, en el DRAE.

Dentro del marco de una investigación más amplia, en la que estudio con detalle diversos aspectos del tratamiento dado a los extranjerismos en las obras académicas, en este trabajo me voy a centrar en los extranjerismos crudos que incluye el nuevo diccionario, es decir, aquellos que, «por no estar adaptados a los patrones gráfico-fonológicos del español» (DRAE 2014: XLVI), presentan el lema en cursiva, siguiendo la dirección abierta por la Real Academia a partir del $D R A E$ (2001). Para ello, recurriré a la comparación con la anterior edición y con las enmiendas para ella adelantadas en el avance ${ }^{3}$, intentando destacar los principales cambios que se producen.

\section{TIPOGRAFÍA Y TIPOS DE ENTRADAS}

Si partimos de los aspectos más externos, observamos que los extranjerismos en cursiva se incorporan, tal como ocurría en 2001, en el cuerpo del diccionario, lo cual, en su momento, ya había sido objeto de críticas por parte de autores que proponían su inclusión en un anexo (cf. Lázaro Carreter 2002 o Martínez de Sousa 2002). Lo sor-

*Este trabajo se enmarca en el proyecto de investigación con referencia FFI2015-65939-P, financiado por el MINECO-FEDER y se ha beneficiado también de una Ayuda de la Xunta de Galicia al Grupo Hispania de la UDC como «Grupo con Potencial de Crecimiento» (ref. GPC2015/028).

${ }^{1}$ Sobre aspectos generales de este nuevo diccionario puede verse Casado Velarde (2015 y Casado Velarde (en prensa).

${ }^{2}$ En el $D E$ (2006) se recogen las novedades aprobadas para la próxima edición del $D R A E$, desde octubre de 2001 a julio de 2006 ( $c f$. www.rae.es). En el avance, las enmiendas incorporadas hasta 2012

${ }^{3}$ Puede verse Sánchez-Martín (2011), para las voces inglesas en el avance.

CRISTINA FERNÁNDEZ BERNÁRDEZ, «Los extranjerismos crudos en el DRAE 2014: Cambios en el diccionario académico», Revista de Lexicografía, XXII (2016), pp. 123-134 ISSN: 1134-4539, e-ISSN: 2603-667. doi: https://doi.org/10.17979/rlex.2016.22.0.3323 
prendente es que la Academia, sin dar ningún tipo de explicación, rectifica con respecto a la decisión tomada en el $D E$, en cuyo prólogo manifestaba:

Adelantándose a lo que hará el DRAE en su edición siguiente, el Diccionario esencial no incluye los extranjerismos registrados en letra cursiva dentro de su nomenclatura general, intercalándolos en la serie de los artículos cuyo lema aparece en redonda. Los sitúa, por el contrario, en una ubicación especial, el APÉNDICE 2 [...] (2006: XX).

Mantiene, eso sí, los dos tipos de entradas que este diccionario presentaba para estos extranjerismos (2006: XX): unos se definen como cualquier entrada del diccionario $\mathrm{y}$ otros remiten a un equivalente o a una adaptación (aproximadamente un tercio de los que he estudiado $)^{4}$.

\title{
3. NÚMERO DE LEMAS EN CURSIVA
}

Un segundo aspecto importante lo constituye el aumento del número de lemas en cursiva. La apertura de la Academia con respecto a la admisión de voces extranjeras en los últimos tiempos ( $c f$. Fernández Bernárdez en prensa a) se hace patente de nuevo en esta edición del DRAE. Si en la 22. ${ }^{a}$ edición su número ascendía a «aproximadamente, 223» (Sabogal 2003: 175; este autor solo ofrece 215), de los cuales 38 ya habían aparecido en ediciones anteriores, pero en letra redonda ( $c f$. Sabogal 2003: 190-192), en 2014 encontramos $539^{5}$. La diferencia de número no se debe solo a la incorporación de nuevas voces, pues muchas aparecían antes de otro modo o con sus lemas en redonda.

Precisamente, el principal incremento viene proporcionado por el cambio que a partir de la Ortografia de 2010 (en adelante OLE) adopta la Academia en el tratamiento de los latinismos. Estos, aunque por tradición académica se recogían en los diccionarios en redonda ( $c f . D R A E$ 2001: XXXV), pasan ahora a tener la misma consideración que los demás extranjerismos y, cuando no estén adaptados, deberán escribirse en cursiva:

\begin{abstract}
Aunque la norma ortográfica académica, desde la Gramática de 1870 hasta la Ortografía de 1999, comenzó recomendando y llegó, más tarde, a prescribir que las voces latinas usadas a menudo en español se sometieran a las mismas reglas de acentuación gráfica que las palabras españolas con el fin de facilitar a todos su correcta lectura, hoy se considera más conveniente tratar los latinismos como los demás préstamos de otras lenguas, de modo que se escribirán con tilde solo cuando se hayan adaptado plenamente al español, esto es, cuando no presenten ningún rasgo ajeno a nuestras convenciones gráfico-fonológicas (OLE: 610).
\end{abstract}

Lo mismo ocurre con las locuciones latinas, que por las mismas razones se incorporaban en redonda y con las tildes pertinentes en el diccionario académico. Se considera ahora que en los textos escritos en español:

\footnotetext{
${ }^{4}$ En Fernández Bernárdez (en prensa b) me detengo en los casos en que se remite a adaptaciones y equivalentes.

${ }^{5}$ Hablo de lemas y no de voces, pues puede darse el caso de dos lemas que se refieran a una misma voz extranjera. Por ejemplo: el lema house reenvía a acid house, forma compleja, s. v. acid; algunas variantes aparecen como lemas diferentes: goulash-gulasch, hippie-hippy, espanglish-spanglish; otras veces hallamos un lema para el acortamiento y otro para la forma completa: heavy metal reenvía a heavy, reality a reality show, mezzo a mezzosoprano, jet2 y jet set presentan ambos idéntica definición perifrástica.
} 
lo más adecuado, en consonancia con el uso culto mayoritario, es que [...] reciban el mismo tratamiento que las de otras lenguas (v. § 2.1.2) y, por tanto, se escriban en cursiva (o entre comillas) y sin acentos gráficos [...] (OLE: 612).

$\mathrm{Al}$ aplicar lo establecido en la $O L E$ al DRAE, tal como se explica en el prólogo de 2014 (XLVI), pasan a mostrarse en cursiva y sin tilde muchos lemas de latinismos y locuciones latinas que antes aparecían en redonda, y que, sumados a los de nueva incorporación, añaden 219 nuevos lemas en cursiva al diccionario.

Por cuestiones de espacio, prescindiré aquí de analizar en detalle las locuciones latinas, pero puedo adelantar que, de un total de 195, solo 29 no aparecían en 2001, si bien algunas se anunciaban como artículos nuevos en el avance (en redonda y con tildes): ad interim, ad limina, ad personam, ad tempus, alea iacta est, a radice, corpore insepulto, gratis et amore, obiter dictum y pro tempore 6 . De modo que son totalmente nuevas 19 locuciones: ab origine, agnus Dei ${ }^{7}$, a limine, aurea mediocritas, carpe diem, contradictio in terminis, ex novo, in absentia, in aeternum, in terminis, in voce, iuris et de iure, lato sensu, manu militari, panem et circenses, per accidens, primus inter pares, vae victis y viva voce.

\section{LOS LEMAS EN CURSIVA. CLASIFICACIÓN}

Dejando aparte las locuciones latinas, hallamos en esta nueva edición del DRAE un total de 344 lemas en cursiva, que clasificaré atendiendo a si son de nueva introducción o si ya aparecían en 2001. Dentro del primer grupo tendré en cuenta si son completamente nuevos o si se anticipaban en el avance. Y dentro del segundo, si se produce algún cambio en el lema o en el cuerpo del artículo. Es preciso tener presente que, tal como se indica en el preámbulo del DRAE 2014, los avances anticipados en la última versión electrónica actualizada «tenían un carácter provisional, no definitivo, lo que supone que no todos ellos se hayan visto necesariamente confirmados en la versión última que aquí se fija» $(\mathrm{X})$.

\subsection{Lemas de nueva introducción}

En el DRAE 2014 encontramos 124 nuevos lemas en cursiva. La gran mayoría corresponden a voces totalmente nuevas (99), pero algunos se anticipaban ya en el avance de la 23. ${ }^{\text {a }}$ edición y otros, un pequeño grupo constituido por 7 lemas, corresponden a extranjerismos crudos cuyas adaptaciones se registraban en 2001.

\subsubsection{Completamente nuevos}

Se trata principalmente de anglicismos ${ }^{8}$ (67 lemas): acid, antidumping, backgammon, backstage, beat, beatnik, birdie, blackjack, blue jean, bogey, boy scout, breakl,

\footnotetext{
${ }^{6}$ Cinco de ellas varían con respecto al avance, pues añaden información etimológica: ad interim, ad limina, ad tempus, obiter dictum y pro tempore.

${ }^{7}$ Remite a la adaptación agnusdéi, en redonda, que sí aparecía en el DRAE 2001.

${ }^{8}$ Para clasificar los extranjerismos según su lengua de origen sigo, en general, la información etimológica dada en el propio DRAE. En ocasiones recurro al Nuevo diccionario de anglicismos (NDA) o a Curell (2004-2005). En Fernández Bernárdez (2017) examino la información etimológica de los extranjerismos crudos en el DRAE 2014.
} 
break2, break dance, bridge, business, cartoon, catch, chill out, coach, country, crack2, crash, eagle, establishment, finger, full, gigabyte, ginseng, hacker, heavy, heavy metal, house, jean, jeep, jumbo, kilobyte, megabyte, performance, playback, playboy, pop art, pub, quad, rafting, reality, reality show, remake, sex shop, sex symbol, shock, show business, showman, show-woman, sketch, spa, spam, squash, swing, terabyte, thriller, tie break, tweed, twist, underground, walkie-talkie y walkman ${ }^{9}$. A los que hay que añadir 2 híbridos español-inglés: colaless y puenting.

Hallamos también 11 galicismos: belle époque, brie, chaise longue, goulash, gulasch, impasse, laissez faire, nonchalance, office ${ }^{10}$, parking ${ }^{11}$ y plié; 2 latinismos: dictum y modus, y 10 voces procedentes de otras lenguas: alemán (land, lied), italiano (mezzo, piccolo, tortellini), japonés (ninja), griego (ethos, pathos), latín científico (rickettsia) y vasco (dantzari). No se hace referencia en el diccionario a la lengua de origen ${ }^{12}$ de doppler, ídish y ndowé.

Incluyo también como lemas nuevos el anglicismo post-it ${ }^{13}$, los galicismos choucroute y luthier y el latinismo fatum, cuyas adaptaciones (pósit, chucrut, lutier y fátum) aparecían como artículos nuevos en el avance, pero como extranjerismos crudos se recogen ahora por primera vez.

\subsubsection{Presentados en el avance}

Los ya anticipados en el avance ascienden a 18, aunque, como ya he dicho, es preciso tener en cuenta que la redacción de los artículos no siempre es idéntica a la del DRAE 2014.

Permanecen inalterados ${ }^{14}$ algo más de la mitad: 4 anglicismos (copyright, jet lag, party, spanglish), 3 galicismos (art nouveau, baguette, boutade) y 3 voces de otras lenguas: italiano (sottovoce), japonés (sushi) y portugués (bossa). Por el contrario, sufren modificaciones 5 anglicismos (bogie, espanglish, off ( en), sparring y stop) y 3 galicismos (amateur, art déco y tour de force $e^{15}$ ). Por lo general se trata de ligeros cambios relacionados con la etimología (se añade en bogie, off ( en), sparring, stop, amateur y se modifica en espanglish) o con la redacción de las definiciones (en todos menos espanglish, que además de la etimología cambia la redonda por cursiva, y sparring).

${ }^{9}$ El $D R A E$ indica que es una marca registrada, pero no de qué lengua procede. El $N D A$ la recoge como anglicismo.

${ }^{10}$ Según se puede leer en el $N D A$ : «En realidad, office es palabra francesa (pronunciada [ofís], pero la creencia de que procede del inglés office ('oficina, despacho') ha generalizado la pronunciación [ófis]» (s. v. office).

${ }_{11}$ Es una voz francesa que procede del inglés.

${ }^{12}$ Tampoco en los acortamientos mezzo y reality, pero en ellos se deduce a partir de las formas completas a las que remiten.

${ }^{13}$ De nuevo, el $N D A$ lo recoge como anglicismo, pero el $D R A E$ solo señala que es una marca registrada.

${ }_{15}^{14}$ Véase apartado 3.2.1.1 para saber exactamente cuándo considero que no hay cambios.

${ }^{15}$ Tour de force se registraba en 2001 como una subentrada de tour. Encontrábamos, además, en el diccionario el lema force, en cursiva, que remitía a tour de force y que ahora desaparece. 


\subsubsection{Recogidos solo como adaptaciones en 2001}

Algunos de los nuevos lemas en cursiva del DRAE 2014 se identifican con extranjerismos que en 2001 se registraban solo como formas adaptadas. En esta nueva edición unas veces el extranjerismo crudo sustituye al adaptado, mientras que en otras convive con él, cumpliendo lo establecido en el prólogo (XLVI): si «se documentan suficientemente en el uso formas crudas y formas adaptadas de un mismo préstamo» se registran ambas, «definiendo la cruda por remisión a la adaptación».

Se produce la sustitución en 3 voces procedentes del italiano (todas pertenecientes al ámbito musical): allegretto, allegro y cantabile, registradas en 2001 como alegreto, alegro y cantábile y que se presentan como artículos enmendados en el avance, aunque la redacción final varía para las dos primeras voces (se añade, en cada caso, una acepción).

La voz vasca abertzale (artículo nuevo en avance ${ }^{16}$ ), los galicismos chapeau y culotte y el anglicismo pallet ${ }^{17}$ conviven con sus respectivas formas adaptadas (aberzale, chapó, culote2 y palé), a las cuales remiten.

\subsection{Lemas que ya aparecían en 2001}

En total ascienden a 220. Distinguiremos aquí entre los lemas que se presentan igual que en la anterior edición del diccionario y aquellos en los que se producen ciertas variaciones. En cualquiera de los casos puede haber, además, cambios en el cuerpo del artículo, en ocasiones recogidos ya en el avance, aunque no siempre coincida este con el resultado final del $D R A E$.

\subsubsection{Sin cambios en el lema}

Son 181 los lemas que no varían con respecto a la edición de 2001. Solo en 75 de ellos el cuerpo del artículo permanece también inalterado.

\subsubsection{Sin cambios en el cuerpo del artículo}

Conviene precisar que no consideraré cambios las modificaciones que afectan a toda la redacción del diccionario, algunas de las cuales, como, por ejemplo, la sustitución de la marca «com.» o por «m. y f.», o de «amb.» por «masc.» o «fem.», se indican en el propio prólogo. Así, no trataré como cambio el uso de abreviaturas en lugar de las expresiones completas o el uso de cifras en lugar de letras en los números. Ni la variación de estilo que supone la repetición del lema en la definición en vez de una expresión perifrástica a él referida (por ejemplo, s. v. fondue: «Conjunto de utensilios para preparar una fondue» (DRAE 2014) / «Conjunto de utensilios para preparar esta comida» (DRAE 2001)). También es sistemática la sustitución de «expresión...» por «locución...», y «Voz eusk.» por «Voz vasca» (esta última ya en la versión en línea de la propia edición de 2001). Finalmente, tampoco tendré en consideración los cambios relativos a mayúsculas y minúsculas en el cuerpo del artículo, derivados de la adaptación a las normas establecidas en la $O L E$.

\footnotetext{
${ }^{16}$ En el avance se propone suprimir el artículo aberzale y abertzale aparece como artículo nuevo, con definición idéntica a la que antes presentaba la voz adaptada. En 2014 experimenta cambios.

${ }^{17}$ Los artículos correspondientes a las formas adaptadas varían con respecto a 2001 en el caso de culote2, en el que se añade una acepción, y en palé, con un importante cambio en la etimología.
} 
Teniendo en cuenta estas matizaciones, encontramos 75 lemas que se pueden incluir en este apartado. La mayoría, concretamente 47, son anglicismos: blazer, boom, bourbon, caddie, camping, casting, catering, clown, cyclo-cross, delicatessen, disc-jockey, dumping, flash, gentleman, gin, gin-fizz, ginger-ale, gin-tonic, green, hall, holding, hooligan, jet1, jet2, jet set, leasing, lifting, light, look, marketing, mass media, offset, ragtime, ranking, reggae, ring, self-service, sheriff, show, software, ${ }^{18}$ sport, $^{19}{ }^{19}$ spot2, standing, tory, watt, windsurf $\mathrm{y}$ windsurfing.

A ellos debemos sumar 13 galicismos (boiserie, boîte, boutique, coulis, coulomb, curie, maître, majorette, motocross, partenaire, rouge, roulotte y souvenir) y 9 voces procedentes de otras lenguas: catalán (rabassa morta), italiano (mozzarella y vendetta), japonés (bushido), latín científico (brassavola), lenca (shunte), náhuatl (cueshte), quechua (pajla) y vasco (ertzaina). No se dan datos sobre la lengua de origen de boshito, cafisho, gauss, guashpira, oersted y shaurire.

Algunas de estas voces se mantienen en el DRAE 2014 a pesar de que en el avance de la 23. a edición se proponía suprimirlas. En concreto, look, self-service, show, spot2, standing, partenaire, rouge y roulotte.

\subsubsection{Con cambios en el cuerpo del artículo}

Más frecuente es que, aunque el lema se mantenga, se produzcan cambios en el cuerpo del artículo; cambios que tienen que ver, generalmente, con la etimología (se modifica, se añaden datos o bien se incluye cuando antes no se hacía), con las marcas técnicas, con la eliminación de variantes ${ }^{20}$ y con las definiciones (varía ligeramente la redacción, se añaden acepciones o se convierte una definición perifrástica en definición por remisión a una adaptación o a un equivalente, o viceversa $)^{21}$. Algunas de estas modificaciones se anticipaban ya en el avance, pero solo en 18 de los 106 lemas que experimentan cambios en el cuerpo del artículo coinciden las versiones de avance y DRAE 2014.

\subsection{Coinciden con el avance}

Se hallan en este caso 12 anglicismos: bungalow, cricket, geisha (etim.), hardware, henry (etim.), hobby, joule (etim.), lobby, maxwell (etim.), newton (etim.), rock y whisky (etim.); 5 galicismos: ampère (etim.), cross (etim.), fondue (etim.), slip (etim.) y troupe, y una voz procedente del afrikáans: apartheid (etim.).

${ }^{18}$ En la versión impresa del $D R A E 2001$ no aparece la marca Inform., que se incorpora ya en la versión en línea y se mantiene en el DRAE 2014.

${ }^{19}$ En este artículo se sustituye la marca geográfica Am. por las marcas Bol., Ec., El Salv., Guat., Hond., Nic., Par., Perú, P. Rico, R. Dom., Ur. y Ven., pero refleja planteamientos generales del diccionario. Según el prólogo, «a partir de esta edición se usa la marca «Am.» (América) para aquellas acepciones con uso atestiguado en catorce países americanos o más» (2014: XLV).

${ }^{20}$ Sobre la presentación de variantes de los extranjerismos crudos en el DRAE 2014 puede verse Fernández Bernárdez (en prensa b).

${ }^{21}$ En cada lema concreto señalaré entre paréntesis los cambios tienen que ver con la etimología, las marcas técnicas y la eliminación de variantes. Los que experimentan variaciones en la definición se marcarán en negrita. En otros trabajos míos se realiza un análisis pormenorizado sobre la etimología de estos lemas, así como de la introducción de adaptaciones y equivalentes y también de las variantes. 


\subsection{No coinciden con el avance}

Se producen cambios en los artículos de 52 anglicismos: baby-sitter, best seller, big bang, blues, body, brandy (etim.), bulldozer, byte, christmas (etim.), crack1, curry, ferry, free lance (etim.), full time, gilbert (etim.), hassio (etim. y desaparece marca Quim. $^{22}$ ), hertz (etim.), hippie, hippy ${ }^{23}$, hockey, input, jacuzzi (etim.), jazz, jogging, lady, living (etim.), lunch (etim.), lycra (etim.), miss (etim.), output, overbooking, panty (etim.), ping-pong (etim. ${ }^{24}$ ), quark ${ }^{25}$, quasar (etim.), rally, rock and roll, rugby, scooter, sexy, sherpa, short (etim.), sponsor (etim.), spot1, spray, sprint, stand, stock, striptease, swahili (etim.), toffee y weber (etim.).

$\mathrm{Y}$ en 22 galicismos: affaire, ballet, becquerel (etim.), beige (etim.), collage, czarda (etim.), déshabillé (etim.), dossier, foie-gras, footing, glamour (etim.), gouache (se añade marca Pint.) ${ }^{26}$, gourmet, mousse, sioux, soufflé, suite ${ }^{27}$, tour (se sustituye marca Mil. por Mar.), tournée, vedette, vichy (etim.) y voyeur. Además de 9 voces procedentes de otras lenguas: italiano (adagio ${ }^{2}$, carpaccio, crescendo (se añade marca Mús.), mezzosoprano (se añade marca Mús.), paparazzi, pizza y pizzicato (etim.)) y náhuatl (maquilishuat y pishishe (etim.)). Y 5 voces cuya lengua de origen no se precisa: gillette (etim. $y$ se elimina la variante gillete), roentgen, shuar, siemens (se sustituye marca Fís. por Electr.) y sievert.

Algunas de estas voces se mantienen en el DRAE 2014, a pesar de que en el avance se propusiese su supresión. Se trata de baby-sitter, christmas, déshabillé, free lance, full time, input, lunch, mousse, output, overbooking, short, spot1, stand, stock, tour y tournée.

\subsubsection{Con cambios en el lema}

En ocasiones encontramos lemas que experimentan variaciones gráficas (ortógráficas o tipográficas) con respecto a la edición de $2001^{28}$. Suman un total de 39 lemas, en cuyos artículos pueden darse a su vez cambios, registrados con anterioridad en el avance o no.

\subsubsection{Cambios ortográficos}

Dos son los tipos de cambios que se observan: los referidos al uso de mayúsculas y los relacionados con las unidades compuestas.

${ }^{22}$ No parece tener mucho sentido la eliminación de esta marca. Posiblemente se trate de una errata.

${ }^{23}$ En realidad, hippie y hippy son variantes. No vemos explicación a que se presenten como lemas independientes, teniendo en cuenta, además, que en 2001 aparecían como un lema doble.

${ }^{24}$ En $D R A E 2001$ aparecía como anglicismo, pero en 2014 se elimina esa información y solo permanece la de «marca registrada». Se registra en el $N D A$.

${ }^{25}$ En quark cambia la etimología con respecto al avance, pero no en relación al DRAE 2001.

${ }^{26}$ Aunque en el avance aparece como «art. emendado», son idénticos el artículo original y el enmendado.

${ }^{27}$ En el avance el lema aparece en redonda, a diferencia tanto del DRAE 2001 como del DRAE 2014.

${ }^{28}$ No incluyo aquí, lógicamente, los cambios que tienen que ver con la desaparición de lemas dobles debidos a la nueva manera de marcar las variantes que el DRAE 2014 explica en sus páginas preliminares. Sobre este tema puede consultarse Fernández Bernárdez (en prensa b). 
En cuanto a los primeros, se registran con minúscula inicial (ya en el avance) 3 lemas de voces procedentes del alemán escritas en 2001 con mayúscula, como corresponde a su lengua de origen: kirsch, kitsch y leitmotiv. En las dos últimas, además, cambia el cuerpo del artículo: se añade en kitsch una acepción y en leitmotiv algunos datos en la información etimológica. Cabe plantearse hasta qué punto es coherente que se acomoden a las normas ortográficas del español desde el momento en que siguen considerándose voces alemanas, pero podría verse como un primer paso hacia su adaptación.

En sentido contrario, el lema Ertzaintza, con mayúscula inicial, aparecía en 2001 en minúscula, contraviniendo las normas de la Ortografía académica vigente en ese momento (1999: 37): «Se escribirán con letra inicial mayúscula [...] los sustantivos y adjetivos que componen el nombre de instituciones, entidades, organismos, partidos políticos, etc.», norma que se conserva actualmente ( $c f r$. OLE: 482-483) y que parece coincidir con la del euskera ( $c f r$. Mujika 2008). En este caso el cambio no se registra en el avance ni se dan variaciones en el cuerpo del artículo.

Un segundo tipo de cambio ortográfico tiene que ver con las unidades compuestas, ${ }^{29}$ en las que puede haber vacilación a la hora de escribirlas en una palabra ortográfica o en dos (separadas mediante guion o no). Cambian, así, los lemas de 4 anglicismos: flashback (en 2001 flash-back); music-hall (en 2001 music hall); sex appeal o sexappeal (en 2001 solo sex-appeal) y top model o top-model (en 2001 solo top-model). Los lemas elegidos en esta nueva edición del DRAE se corresponden con las formas etimológicas registradas en el NDA. Varía también el cuerpo del artículo en flashback y top model, donde hay cambios en la definición. El avance solo registra las modificaciones referidas a flashback, tanto del lema como del cuerpo del artículo.

\subsubsection{Cambio de redonda a cursiva}

Algunos de los extranjerismos registrados en cursiva en 2014 aparecían en la edición de 2001 en redonda, a pesar de presentar rasgos gráficos o fónicos ajenos a las normas del español. Por la manera en que se trata la etimología de estas voces en 2001, parece que la RAE las considera formas adaptadas al español, con las repercusiones que esto tendría en su pronunciación ${ }^{30}$. Este reajuste supone, pues, en principio, una mejora en la selección de los extranjerismos crudos, además de un aumento del número de lemas en cursiva.

Al mismo tiempo, también en estas voces pueden darse cambios en el cuerpo del artículo, que afectan a la etimología, a las definiciones y a las marcas técnicas ${ }^{31}$.

El grupo más numeroso de lemas que pasan de redonda a cursiva lo constituyen, lógicamente, los latinismos (21 lemas), con respecto a los cuales, como hemos visto, la RAE ha cambiado su planteamiento. Así, ahora aparecen en cursiva absit (etim.), apud

\footnotetext{
${ }^{29}$ Sobre estas unidades me extiendo más en otros trabajos relacionados con esta investigación: Fernández Bernárdez (en prensa b) y Fernández Bernárdez (2017).

${ }^{30}$ El cambio de redonda a cursiva debería implicar, además, un cambio en la etimología de estas voces, pues pasan a «ser» voces extranjeras en lugar de «proceder de» una voz extranjera. Así sucede con los latinismos absit, apud, confer, ibidem, idem, nequaquam, nominatim, passim, quadrivium, summum, transeat, trivium y valgus, y las voces decrescendo, grill, jiu-jitsu y pidgin.

${ }^{31}$ Remito a la nota 19 para ver cómo se mostrarán esos cambios.
} 
(etim.), confer (etim.), cunnilingus (etim.), exequatur (etim.), ibidem (etim.), idem (etim.), mellitus (etim.), nequaquam (etim.), nominatim (etim.), nullius (etim.), passim (etim.), quadrivium (etim.), quater, quinquies, quorum (etim.), summum (etim.), transeat (etim.), trivium (etim.), valgus (etim.) y vide, que en 2001 y en el avance se presentaban en redonda y con las tildes pertinentes. Excepto en el caso de vide, donde el cambio afecta únicamente al lema, en todas estas voces se producen también variaciones en el cuerpo del artículo. En idem, el DRAE 2014 mantiene el lema en redonda y con tilde y añade otro lema en cursiva ${ }^{32}$.

A su lado encontramos otras 10 voces que experimentan este cambio (aunque solo una, pidgin, se presenta en el avance). En todas ellas se producen también cambios en el cuerpo del artículo. Se trata de 3 anglicismos: grill (etim.), jiu-jitsu (etim.) y pidgin (etim. $y$ se añade marca Ling.); un galicismo: tell (etim.); un italianismo: decrescendo ${ }^{33}$ (etim.); una voz procedente del hebreo: sabbat; otra del lat. científico: wellingtonia (etim.) y 3 cuya lengua de origen no se precisa: mbayá, tawahka y twahka.

\section{LEMAS EN CURSIVA EN 2001 QUE DESAPARECEN EN 2014}

Algunas voces cuyo lema estaba en cursiva en la anterior edición del DRAE desaparecen en esta nueva edición: bien porque se eliminan totalmente, bien porque son sustituidas por su adaptación al español.

Entre los que desaparecen por completo se hallan 3 anglicismos (dancing, gang y speech), 2 galicismos (chartreuse y force) y un germanismo (Bock). Solo para los anglicismos y para force se proponía en el avance la supresión. Llama la atención la eliminación de Bock: en el avance se presentaba como artículo enmendado, cambiando la mayúscula inicial por minúscula, como hemos visto que sucede con otras voces procedentes del alemán. También desaparece el lema force (que remitía a tour de force, $s . v$. tour), pero es sustituido por un nuevo lema en cursiva: tour de force.

Más abundantes son las voces que permanecen en el diccionario como formas adaptadas. Las adaptaciones pueden ser más o menos complejas: asimilación al español de grafías ajenas (ciertos grupos consonánticos, consonantes duplicadas, $y$ con valor vocálico en interior de palabra...), adición de tilde, o, simplemente, adaptación de la pronunciación a la grafía, sin que existan cambios en esta última.

Encontramos en este grupo 10 anglicismos: gong (> gong), gospel (> góspel), handicap ( $>$ hándicap), junior $\left(>\right.$ júnior $\left.^{34}\right)$, ketchup ( $>$ kétchup), klystron ( $>$ klistrón), paddle ( $>$ pádel), punk ( $>$ punk), topless ( $>$ toples) y western ( $>$ wéstern). Además de 2 galicismos: forfait (>forfait) y quiche (> quiche). Y 3 voces procedentes de otras len-

${ }^{32}$ Estos lemas comparten la definición («el mismo, lo mismo»), aunque para el extranjerismo crudo se reserva la aclaración «U. generalmente abreviado en las citas para representar el nombre del autor últimamente mencionado, y en las cuentas y listas, para denotar diferentes partidas de una sola especie». Las expresiones complejas quedan repartidas entre ambos: al lema en cursiva se adscribe idem per idem y al otro ídem de ídem.

${ }^{33}$ Resultaba particularmente incoherente que este lema apareciese en redonda, teniendo en cuenta que crescendo se registraba en 2001 en cursiva como «Voz it.».

${ }^{34}$ Posiblemente debido a un error, en la versión en línea del DRAE 2001 no se encuentra el anglicismo junior, que sí aparece, en cursiva, en la versión impresa. 
guas: italiano: ossobuco (> osobuco); quechua: mashca ( $>$ máchica) y chino: yang $(>$ yang). Finalmente, 6 voces cuya lengua de origen no se indica: alzheimer ( $>$ alzhéimer), angstrom (> ángstrom), balimbing (> balimbín), cicca (> cica), kilohertz (> kilohercio), megahertz (> megahercio).

La mayor parte de estos cambios se anuncian ya en el avance. Son nuevos únicamente los referidos a angstrom, balimbing, cicca, handicap, junior, kilohertz, mashca, megahertz y yang. Por otra parte, en el DRAE 2001 los extranjerismos crudos balimbing, cicca, mashca, megahercio y kilohercio convivían ya con la forma adaptada.

Muchos de los cambios que introduce el DRAE en cuanto a la elección de la cursiva y la redonda producen cierto desconcierto. A simple vista, parece dicífil entender el paso a la redonda de alzheimer, angstrom, gong, ketchup, punk, western o yang, pues estas grafías parecen ajenas al español. Y, en sentido contrario, el paso de la redonda a la cursiva de, por ejemplo, muchos latinismos que no parecen ajenos ni a la grafía ni a la pronunciación el español ${ }^{35}$.

\section{CONCLUSIONES}

El cambio producido en la edición de 2001 del $D R A E$ con respecto a la introducción de extranjerismos en cursiva se mantiene en la nueva edición del diccionario, dejando a un lado la solución, ofrecida por la RAE en el $D E$ (2006), de incluir los extranjerismos crudos en un apéndice.

En muchas ocasiones, aproximadamente en un tercio de los casos estudiados, se ofrecen definiciones que remiten a una adaptación o a un equivalente. Se documenta así, por un lado, la convivencia de forma cruda y forma adaptada de un mismo extranjerismo, en un período en que este todavía se encuentra en una etapa de integración al español. Por otro, se constata el hecho de que el uso puede acabar imponiendo la entrada de un extranjerismo, pese a la existencia de un equivalente en español.

Es significativo el aumento del número de lemas en cursiva, debido en parte a la adaptación del diccionario a las normas de la $O L E$ con respecto a los latinismos, pero también a la apertura que en los últimos años caracteriza a la Academia con respecto a la admisión de extranjerismos. Más abierta incluso en la redacción definitiva del diccionario que en el avance, ya que muchas voces para las que este proponía la supresión (por innecesarias, al existir un equivalente español) finalmente perviven en el diccionario. Sin contar los latinismos, que en 2001 aparecían en redonda, tenemos 124 nuevos extranjerismos crudos en el DRAE 2014, de los cuales solo 18 se anticipaban en el avance.

En cuanto a las voces en cursiva que aparecían en $2001^{36}$, 181 se mantienen con el lema sin variaciones y 8 presentan algún cambio ortográfico. En la mayoría de ellas, sin embargo, se producen modificaciones en el cuerpo del artículo, que afectan a la etimología, las marcas, las variantes o la definición. Además, en 2014 desaparecen 6 voces, 4 de las cuales se proponían ya para supresión en el avance. Al mismo tiempo,

${ }^{35}$ Dado que las explicaciones de las páginas preliminares del diccionario no dan pistas suficientes para entender algunos casos, parece necesaria una reflexión sobre este aspecto, que abordo en otros trabajos relacionados con esta investigación: Fernández Bernárdez (2017 y en prensa b).

${ }^{36}$ Tomo como referencia a Sabogal (2003), que comenta 215 voces en cursiva del DRAE 2001. 
pasan a considerarse extranjerismos adaptados, con cambio de la cursiva a la redonda (a lo que se añaden, cuando es necesario, otras modificaciones que permiten su adaptación), 21 lemas.

Aparecen ahora en cursiva, además de los latinismos (que se adaptan a las normas de la $O L E), 10$ lemas que en 2001 se presentaban en redonda, aplicando así de un modo más coherente el principio de emplear la cursiva para los lemas que no se ajustan a las normas gráficas o de pronunciación del español. En todos ellos se mejora también el cuerpo del artículo.

\section{REFERENCIAS BIBLIOGRÁFICAS}

CASAdo Velarde, Manuel (2015): La innovación léxica en el español actual, Madrid, Síntesis.

CASADO Velarde, Manuel (en prensa): «La innovación léxica en el DRAE 2014», Español Actual.

Curell Aguilá, Clara (2004-2005): Presencia del francés en el español peninsular contemporáneo, Universidad de la Laguna. En línea en ftp://tesis.bbtk.ull.es/ ccssyhum/cs207.pdf.

DE. ReAl ACADEMIA EsPañola (2006): Diccionario esencial de la lengua española, Madrid, Espasa Calpe. En línea en http://www.rae.es/recursos/diccionarios/desen.

DEA. Manuel SeCo, Olimpia de ANDRÉS y Gabino RAmos (2011): Diccionario del español actual, 2. ${ }^{\mathrm{a}}$ edición, Madrid, Aguilar.

DPD. Real ACADEMia Española y Asociación de ACAdEMias de la Lengua EspañoLA (2005): Diccionario panhispánico de dudas, Madrid, Santillana. En línea en http://www.rae.es.

DRAE 2001. REAL ACADEMIA EsPañola (2001): Diccionario de la lengua española, Madrid, Espasa Calpe, 22. ${ }^{\mathrm{a}}$ ed. En línea en http://rae.es. (DRAE 2001). Avance de la 23. ${ }^{\text {a }}$ ed. en línea en http://rae.es.

DRAE 2014. ReAl ACADEMIa Española y Asociación De ACADEMias DE LA LENGUA ESPAÑOLA (2014): Diccionario de la lengua española, Barcelona, Espasa, 23. ${ }^{a}$ ed.

FERNÁNDEZ BERNÁRDEZ, Cristina (2017): «La información etimológica de los extranjerismos crudos en la nueva edición del DRAE», Círculo de Lingüística Aplicada a la Comunicación, 69, pp. 136-159.

FERNÁNDEZ BERNÁRDEZ, Cristina (en prensa a): «Tratamiento de los extranjerismos en las últimas obras académicas», en Munere bene functo. Homenaxe galega a Álvaro Porto, A Coruña, Universidade da Coruña.

FERNÁNDEZ BERNÁRDEZ, Cristina (en prensa b): «Los criterios aplicados en el DRAE 2014 a los extranjerismos crudos. Revisión crítica», Rilce.

LÁZARO CARRETER, Fernando (2002): «El neologismo en el diccionario», Discurso en la presentación de la Escuela de Lexicografía Hispánica, en línea en www.virtualcervantes.com.

MarTíneZ De SousA, José (2002): «Neologismos en el Diccionario de la Academia (edición de 2001)», en Actas del I Congreso Internacional «El Español, Lengua de Traducción», Almagro, pp. 175-185. 
MUJIKA, Alfontso (2008): Letra larriak erabiltzeko irizpideak (Criterios para el uso de las mayúsculas). En línea en http://www.hezkuntza.ejgv.euskadi.eus/r43euskcont/es/contenidos/informacion/dih/es_5490/adjuntos/estilo_liburua/letra_larri ak.pdf?_ga=1.68891857.1715018181.1426072679).

NDA. Félix RodRíguez GonzÁlez, Félix, dir., y Antonio LiLlo (1997): Nuevo diccionario de anglicismos, Madrid, Gredos.

OLE. Real ACADEMia Española y Asociación de ACADEMias de la Lengua Española (2010): Ortografia de la lengua española, Madrid, Espasa. En línea en http://aplica.rae.es/orweb/cgi-bin/buscar.cgi.

Real ACAdEMia Española y Asociación de ACAdemias de la Lengua Española (2009): Nueva gramática de la lengua española, Madrid, Espasa.

Real ACADEMIa ESPaÑola y Asociación de ACADEMias DE LA LENGUA ESPañola (2011): Nueva gramática de la lengua española. Fonética y fonología, Barcelona, Espasa.

SABOgal CÁRdenas, Cleóbulo (2003): «Extranjerismos en el Diccionario de la lengua española», Boletín de la Academia Colombiana, 54, 221-222, pp. 175-194.

SÁNCHEZ MARTín, Francisco Javier (2011): «El trabajo de la Real Academia Española en el avance de la 23 . $^{\text {a }}$ edición del Diccionario de la lengua española: las voces inglesas», Lexis, XXXv, 1, pp. 143-161.

Título

«Los extranjerismos crudos en el DRAE 2014: Cambios en el diccionario académico»

«Rough foreignisms in the new edition of DRAE (2014): Changes in the academic dictionary»

Fecha de recepción: 15/09/2016 Fecha de aceptación: 09/12/2016

Resumen: En este artículo se realiza una revisión crítica del tratamiento dado en la 23.a edición del DRAE (2014) a los extranjerismos crudos, es decir, aquellas voces que, por no atenerse a las normas gráfico-fonológicas del español, se presentan en la obra académica con lemas en cursiva. Se comparan, cuando es posible, con la anterior edición del DRAE y con el avance de la 23. ${ }^{\mathrm{a}}$, en un intento de observar qué criterios se aplican y si se hace coherentemente.

Palabras clave: lexicografía, DRAE 23. a edición, extranjerismos crudos, cambios.

Abstract: This paper offers a critical review regarding the presence and consideration of 'extranjerismos crudos' (rough foreigisms) in the $23^{\text {rd }}$ edition of DRAE (2014); namely, those words that do not follow the spelling and phonetic rules of the Spanish language and appear, for that reason, as lemmas in italics in the academic dictionary. The previous edition of DRAE and the preview for the $23^{\text {rd }}$ edition are compared, when possible, with the aim to observe the criteria employed and determine if said criteria has been applied in a proper and consistent manner.

Keywords: lexicography, 23rd edition of the DRAE, rough foreigisms, changes. 